\title{
CONSENSORS: A Neural Network Framework for Sensor Data Analysis
}

\author{
Burkhard Hoppenstedt, Rüdiger Pryss, Klaus Kammerer, and Manfred \\ Reichert \\ Institute of Databases and Information Systems, Ulm University, Germany
}

\begin{abstract}
Machine breakdowns in industrial plants cause production delays and financial damage. In the era of cyber-physical systems, machines are equipped with a variety of sensors to monitor their status. For example, changes to sensor values might indicate an abnormal behavior and, in some cases, detected anomalies can be even used to predict machine breakdowns. This procedure is called predictive maintenance, which pursues the goal to increase machine productivity by reducing down times. Thereby, anomalies can be either detected by training data models based on historic data or by implementing a self-learning approach. In this work, the use of neural networks for detecting anomalies is evaluated. In the considered scenarios, anomaly detection is based on temperature data from a press of a machine manufacturer. Based on this, a framework was developed for different types of neural networks as well as a high-order linear regression approach. We use the proposed neural networks for restoring missing sensor values and to improve overall anomaly detection. An evaluation of the used techniques revealed that the high-order linear regression and an autoencoder constitute best practices for data recovery. Moreover, deep neural networks, especially convolutional neural networks, provide the best results with respect to overall anomaly detection.
\end{abstract}

Keywords: Anomaly Detection · Sensor Data Recovery

\section{Introduction}

In modern industrial plants, a rising number of sensors offers advanced opportunities for automatically detecting machine faults. In this context, machinelearning methods have proven to be beneficial [1]. Although first models related to neural networks were already introduced in the 1940s [2], their usage in an industrial context only increased significantly during the last years. Thereby, data storage capabilities constitute an important factor for the development of neural networks as large training data sets usually enhance the overall outcome. Furthermore, the developments in the field of deep learning enable a better readability of neural networks. This improvement is achieved by abstracting information into hierarchically ordered layers. In this work, we apply neural networks techniques to an industrial data set, provided by an automotive press company. 
More precisely, the data set contains temperature sensor data from three engines in a press and the difference between engine temperatures is used to represent anomalies (cf. Fig. 1, left). These data have been collected over a year of operation before a machine breakdown occurred. With the help of machine learning techniques, we tackle two use cases: First, we aim to recognize anomalies. Second, we try to recover lost (i.e., missing) sensor data. Regarding the first use case, the results can be utilized in advanced information systems for the provision of alerts and condition monitoring. Following the idea that anomalies occur more frequently before a breakdown, they can be used as input for predictive maintenance. Concerning the second use case, data recovery is useful to improve overall data quality and, therefore, enhance data analytics approaches.
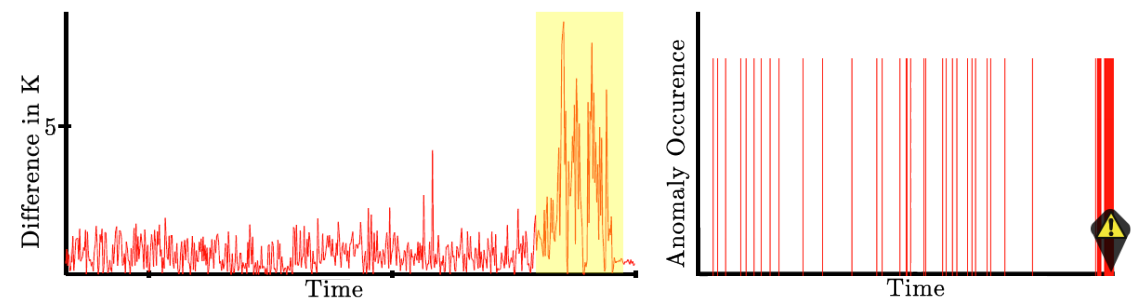

Fig. 1. Extract from Measured Sensor Data (left) and Increasing Occurences of Anomalies before a Breakdown (right)

\section{Data Set \& Evaluation}

The data set stems from a machine manufacturer and consists of values from a press line with six presses. It was recorded over a period of one year, using a sampling rate of one measurement per minute. At the end of this year, a breakdown of one press occurred (cf. Fig. 1, right part). To apply methods of supervised learning, the data points are labeled. Thereby, a single data point was marked as anomaly if any of the three engine temperature differences was greater than 5 Kelvin (K). Then, a data section of 120 data points (i.e., each with three temperature values) was used as the input for the neural networks. Note that this corresponds to a 2-hour measurement. Furthermore, an input is labeled as anomaly when it contains at least 105 single data points that are regarded as an anomaly. Using this approach, we train the neural network to recognize anomaly patterns. Hereby, we recognize exceeded temperature thresholds as well as the time series structure. Next, the data set is split up into training and test data. As the anomalies are underrepresented in the whole data set (cf. Tab. 1), test data is randomly generated by $1 \%$ normal input data and $20 \%$ of the anomaly input data.

The proposed framework hierarchy is shown in Fig 3. It is optimized for sensor data and includes various types of neural networks. To calculate the precision for 
Table 1. Distribution of the Data Set

\begin{tabular}{|c|c|c|c|c|}
\hline ID & Data Label & Data Set Size & \multicolumn{2}{|l|}{ Percentage of Parent } \\
\hline 1 & All Data & 524162 & & \\
\hline 2 & Normal Data & 518095 & $98.8 \%$ of & 1 \\
\hline 3 & Anomaly Data & 6067 & $1.2 \%$ of & 1 \\
\hline 4 & Training Data Normal & 512915 & $99.0 \%$ of & 2 \\
\hline 5 & Training Data Anomaly & 4854 & $80.0 \%$ of & 3 \\
\hline 6 & Test Data Normal & 5180 & $1.0 \%$ of & 2 \\
\hline 7 & Test Data Anomaly & 1213 & $20.0 \%$ of & 3 \\
\hline
\end{tabular}

the use case Sensor Value Restoration, the mean derivation of the expected value is used as evaluation criterion. For the use case Anomaly Detection, the number of unrecognized anomalies (false negative) as well as the number of wrongly discovered anomalies (false positives) are used to calculate the $F_{1}$-score. These two evaluation criteria are shown in Fig. 2.
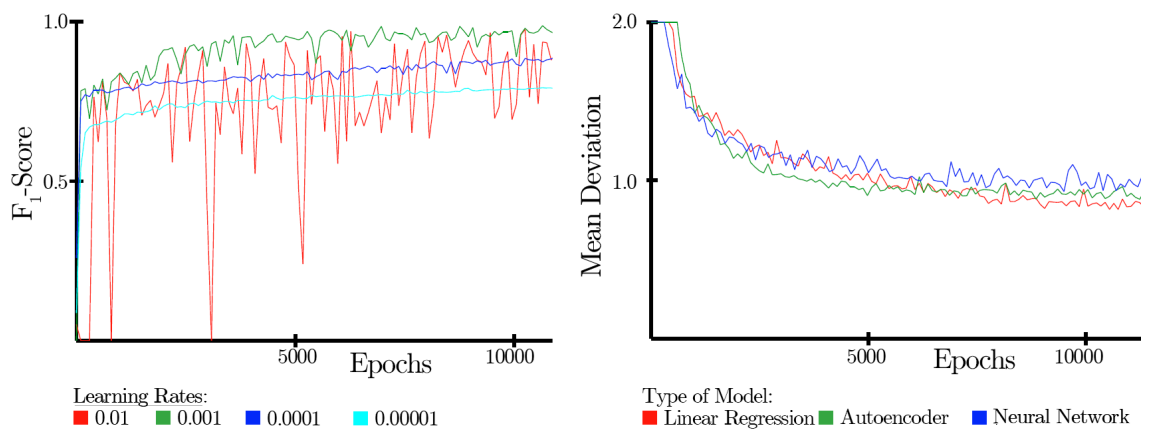

Fig. 2. $F_{1}$-Scores (left) and Mean Deviation (right)

In Fig 3, the most promising results of the framework are shown. The autoencoder (AE) performed well for recovering sensor data, but its variants denoisingautoencoder (DAE) and compression-autoencoder (CAE) achieve a low $F_{1}$-score. In contrast, a fully connected neural network (FCNN) performed better for anomaly detection than sensor value recovery. Note that the order of the best linear regression (LR) was four. Finally, convolutional neural networks are promising for anomaly detection.

\section{Summary and Outlook}

The presented framework performs well on the shown data set. It restores missing temperature values with an average deviation of less than 1 Kelvin and recognizes anomaly patterns reliably. Overall, the main advantages of the framework 
are as follows: First, due to the hierarchical class structure, it is possible to switch between performance and reliability. High-level networks (e.g., the autoencoder) need more computation time, but achieve better results. Second, the framework is designed for sensor data and allows to select a time window to be evaluated. However, the neural networks need high computation power and, for supervised learning, a pre-labeled data set is required. In future work, recurrent neural networks [3] may provide further improvements, as they include previous decisions into a classification. Other approaches dealing with the application of neural networks for sensor data exist. For example, [4] discusses an application of neural networks in a motor system, where vibration signatures are analyzed with the help of neural network agents. Next, [5] reached $F_{1}$-scores up to 0.987 , using linear autoencoders to detect anomalies in labeled audio databases. However, to our best knowledge, the presented framework combined with data of a press manufacture illustrate new insights.

\begin{tabular}{|c|c|c|c|c|c|c|c|}
\hline \multirow[b]{2}{*}{ Interface: ITransformator } & \multirow[b]{2}{*}{ Perceptron } & \multirow{4}{*}{$\begin{array}{l}\text { Model } \\
\mathrm{AE}\end{array}$} & \multirow{4}{*}{\begin{tabular}{|l|} 
Info \\
(Distortion) \\
0.00 \\
0.01 \\
$\mathbf{0 . 0 5}$ \\
\end{tabular}} & \multirow{4}{*}{\begin{tabular}{|l}
$\operatorname{std}(\mathbf{K})$ \\
\\
0.95 \\
0.94 \\
$\mathbf{0 . 9 3}$
\end{tabular}} & \multirow{4}{*}{\begin{tabular}{|l|} 
std $>\mathbf{2 k}$ \\
\\
$11.5 \%$ \\
$10.7 \%$ \\
$\mathbf{1 0 . 5 \%}$
\end{tabular}} & \multicolumn{2}{|c|}{\begin{tabular}{|l|l|} 
Model & F1 \\
\end{tabular}} \\
\hline & & & & & & \begin{tabular}{|l|} 
PER. \\
\end{tabular} & 0.70 \\
\hline & & & & & & DAE & 0.70 \\
\hline BasicNetwork & LinearRegression & & & & & CAE & 0.72 \\
\hline & & $J \mathrm{P}$ & (Order) & & & & \\
\hline NeuralNetwork & Autoencoder & & 2 & 0.87 & $9.8 \%$ & & 0.983 \\
\hline & & & $\begin{array}{l}3 \\
4\end{array}$ & $\begin{array}{l}0.80 \\
0.79\end{array}$ & $8.4 \%$ & $\| \mathrm{CN}(1,0)$ & 0.988 \\
\hline Specialized & Regenerating & & 5 & 0.78 & $8.6 \%$ & $\mathrm{CN}(2,1)$ & 0.993 \\
\hline ConvolutionalNetwork & Autoencoder & FCNI & & 102 & $126 \%$ & $\mathrm{CN}(2,2)$ & 0.972 \\
\hline
\end{tabular}

Fig. 3. Framework (1), Recovery Results (2) and Anomaly Detection Results (3)

Altogether, neural networks are able to adapt to production changes by adjusting their weights. Moreover, they are robust to irrelevant noise, as they are trained to discover only relevant sensor patterns. Therefore, the application of neural networks is promising in the fields of sensor data recovery and anomaly detection.

\section{References}

1. Zhao, R. et al.: Deep learning and its applications to machine health monitoring: A survey. arXiv:1612.07640. (2016)

2. Schmidhuber, J.: Deep learning in neural networks: An overview. Neural networks, 61, 85-117 (2015)

3. Gugulothu, N. et al.: Predicting Remaining Useful Life using Time Series Embeddings based on Recurrent Neural Networks. arXiv:1709.01073. (2017)

4. Li, B. et al.: Neural-network-based motor rolling bearing fault diagnosis. IEEE transactions on industrial electronics 47, 1060-1069 (2000)

5. Marchi, E. et al.: Deep recurrent neural networkbased autoencoders for acoustic novelty detection. Computational intelligence and neuroscience (2017) 